Gdańsk 2018, Nr. 38

Agnieszka K. Haas

Uniwersytet Gdański, Wydział Filologiczny /

Universität Gdańsk, Philologische Fakultät

https://doi.org/10.26881/sgg.2018.38.03

\title{
Zur Unverständlichkeit von Chiffren und Hieroglyphen der Natur in der Prosa der Frühromantik
}

Im vorliegenden Beitrag gilt die Aufmerksamkeit dem Topos vom Buch der Natur in der deutschen Prosa der Frühromantik und seinem Verhältnis zu rhetorischen Figuren, die auf die transzendente Quelle der Natur verweisen. In den Vordergrund rückt die von den romantischen Dichtern betonte Unmöglichkeit, die Chiffren und Hieroglyphen der Natur zu enträtseln. Ursprünglich bedeutete das Buch der Natur eine an den Menschen gerichtete chiffrierte Mitteilung Gottes bzw. des Geistes. Die Romantiker betrachten die Natur als geheimnisvolle Chiffre, die in zunehmendem Maße unverständlicher wird. Die Fragestellung im Artikel lautet: Mithilfe von welchen rhetorischen Mitteln wird der geistige Charakter der Natur ausgedrückt und in welchem Zusammenhang steht der genannte Topos mit dem Problem des beschränkten Sprach- und Erkenntnisvermögens in der Literatur um 1800.

Schlüsselwörter: Buch der Natur, Frühromantik, Friedrich von Hardenberg (Novalis), Wilhelm Heinrich Wackenroder, Rhetorik

The present article The incomprehensibility of ciphers and hieroglyphs of nature in the German prose of early Romanticism discusses the Book of Nature metaphor in the German literature of early Romanticism in its relation to the rhetorical devices which represent the spiritual character of nature. Particular attention was devoted to the problem of the impossibility of understanding the cipher and hieroglyphs of nature claimed by Romantic poets. The Book of Nature was understood in earlier rhetoric as God's message to human beings. At the beginning of the $19^{\text {th }}$ century, nature was considered as a mysterious cipher becoming less and less understandable. The article raises the question as to what kind of rhetorical devices show the spiritual character of nature and in what way the topos is used to show the problem of limited linguistic and cognitive capacity in the literature of around 1800.

Keywords: Book of Nature, early Romanticism, Friedrich von Hardenberg (Novalis), Wilhelm Heinrich Wackenroder, rhetorical devices

\section{Rhetorik (in) der Romantik}

Einen Traditionsumbruch in der Rhetorikgeschichte brachte gerade die ,rhetorikverach-

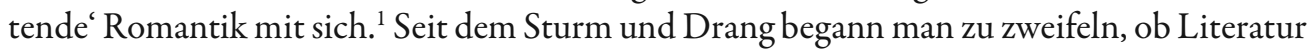

1 Helmut Schanze, Romantische Rhetorik, in: ders. (Hrsg.), Romantik-Handbuch, Stuttgart 1994, S. 339. 
„die kasuistischen Vorschriften“ der Rhetorik benötigt ${ }^{2}$ und ob sie imstande ist, semantische Probleme der Psychologie oder Philosophie richtig auszudrücken. Ihre Regeln scheinen vor allem in Bezug auf die literarische Behandlung metaphysischer Themen unanwendbar zu sein. Das Verhältnis der Romantiker zur Rhetorik ist nichtsdestoweniger alles andere als eindeutig, und obwohl Kant sie als eine zweckorientierte Kunst verstand, die die Menschen durch den schönen Schein hintergehen will, ${ }^{3}$ wurde sie von den Romantikern nicht ganz abgelehnt. Einerseits scheinen Romantik und Rhetorik sich auszuschließen, ${ }^{4}$ andererseits gilt diese Epoche als „Zweite Renaissance “" und Zäsur in der Rhetorikgeschichte. ${ }^{6}$

Die neuere Forschung erkennt in der Romantik Kontinuitäten der Aufklärung, wie zum Beispiel das Aufgreifen des uralten Topos vom Buch der Natur. Auch die rhetorischen Figuren, die die Romantiker in Anspruch nehmen, um die Chiffren und Hieroglyphen der Natur zu schildern, sind eher konventionell.

Im vorliegenden Beitrag soll gezeigt werden, wozu rhetorische Stilmittel in der Frühromantik dienen und welche von ihnen bei der Darstellung der Natur-Topik vorkommen, die auf das Problem der Begrenztheit von Erkenntnis und Sprache hinweisen. Dabei scheinen zwei Bereiche der Rhetorik von Relevanz zu sein: Die „Durchdringung der Literaturen mit [...] rhetorischer Poetik“ und die Betrachtung der Rhetorik als „Anordnungsinstrument der Topik“.7

\section{Das Buch der Natur und seine literarischen Kontinuitäten}

Eine umfassende Geschichte des Topos würde den Rahmen der vorliegenden Skizze sprengen. Obwohl sie an mehreren Stellen präsentiert wurde, ${ }^{8}$ ist es an dieser Stelle angebracht, einige ihre Etappen zu nennen, die mit dem frühromantischen Weltschriftkonzept zusammenhängen.

In der neuplatonisch-christlichen Tradition war das Buch der Natur (lat. liber naturae) neben der Heiligen Schrift eine Quelle der Erkenntnis Gottes. Sowohl die Heilige Schrift als auch wahrnehmbare Phänomene der Außenwelt galten als eine an den Menschen gerichtete

2 Rüdiger Campe, Umbrüche und Wandlungen der Rhetorik, in: Horst Albert Glaser, György Mihály Vajda (Hrsg.), Die Wende von der Aufklärung zur Romantik 1760-1820: Epoche im Überblick, Amsterdam, Philadelphia 2001, S. 589-612, hier: S. 589.

3 Jochen A. Bär, Romantik [Artikel], in: Gert Ueding, Gregor Kalivoda u.a. (Hrsg.), Historisches Wörterbuch der Rhetorik, Bd. 8, Tübingen 2007, S. 333-362, hier S. 338-339.

4 Helmut Schanze, Romantik und Rhetorik, Rhetorische Komponenten der Literaturprogrammatik um 1800, in: ders. (Hrsg.), Rhetorik. Beiträge zu ihrer Geschichte in Deutschland vom 16.-20. Jahrhundert, Frankfurt a.M. 1974, S. 126-144, hier: S. 126. Zit. nach: Peter D. Krause, Unbestimmte Rhetorik. Friedrich Schlegel und die Redekunst um 1800 [Reihe: Rhetorik-Forschungen, hrsg. von Joachim Dyck, Walter Jens, Gert Ueding, Bd. 14], Tübingen 2001, S. 13.

5 Helmut Schanze, Romantische Rhetorik, S. 337.

Vgl. Peter D. Krause, Unbestimmte Rhetorik, S. 13.

Die erste Kategorie - die Rhetorik der Klassikerlektüre und der Stilübung - spielt hier kaum eine Rolle. Vgl. Rüdiger Campe, Umbrüche und Wandlungen der Rhetorik, S. 589-590.

8 Henri Veldhuis, Ein versiegeltes Buch. Der Naturbegriff in der Theologie J.G. Hamanns (1730-1788). Aus dem Niederländ. übers. von Renate Drewes-Siebel, Berlin, New York 1994, S. 27. In der Anmerkung 41 (S. 27) findet man auch eine Bibliographie zu diesem Thema. 
Mitteilung Gottes. Zur Entstehung der Metapher hat das von Augustinus eingeführte Gleichnis ${ }^{9}$ beigetragen. In seinen Schriften wird jedoch der Begriff liber naturae nicht erwähnt. ${ }^{10}$ Zuerst hat sich der Terminus in den mystisch-philosophischen Spekulationen und dann im allgemeinen Sprachgebrauch verankert. Den mittelalterlichen Theologen diente die Metapher dazu, die Sinnhaftigkeit der Erscheinungswelt zu legitimieren ${ }^{11}$ und die Offenbarung durch die Schöpfung zu versinnbildlichen. ${ }^{12}$

Der Begriff, Buch der Natur ' wurde in der Frühen Neuzeit in naturwissenschaftlichen Schriften und Spekulationen der Naturmystik verwendet, ${ }^{13}$ in denen er einen neuen Aufschwung erfuhr. Galileo Galilei gebraucht ihn in Bezug auf mathematische Regeln. Die Naturphilosophie, Zahlenlehre und jüdische Kabbala verknüpften die Mathematik mit der Sprache und Magie - vor allem dieses Konzept wurde in der Romantik mehrmals aufgegriffen. Noch im 17. Jahrhundert glaubte man in der Natur zwei Bücher, „Religion und Naturwissenschaft", zusammenzuführen und dadurch Glauben und Wissen zu harmonisieren. ${ }^{14} \mathrm{Im}$ philosophischen Diskurs verwenden die Metapher Bacon, Descartes oder Leibniz. ${ }^{15}$ Anfang des 18. Jahrhunderts liegt der Topos vom Buch der Natur in der Physikotheologie vor, die die Theologie mit der Naturlehre vereinbaren wollte. Ihre Spekulationen wurden von vielen Rationalisten abgelehnt, ${ }^{16}$ aber in der Literatur wurden sie von vielen Autoren erwähnt, wie

9 Andreas Speer, Die entdeckte Natur. Untersuchungen zu Begründungsversuchen einer, scientia naturalis‘ im 12. Jahrhundert, Leiden, New York, Köln 1995, S. 30.

10 Gerhard Sauder, Aufklärerische Bibelkritik und Bibelrezeption in Goethes Werk, in: „Goethe-Jahrbuch“ 2001, Bd. 118, S. 108-125, hier: S. 108. Das Vorkommen der Metapher wurde bereits in der voraugustinischen Zeit belegt. Vgl. Günter Bader, Melancholie und Metapher: eine Skizze, Tübingen 1990, S. 61.

11 Monika Schmitz-Emans, Buch [Artikel], in: Günter Butzer, Joachim Jacob (Hrsg.), Metzler Lexikon literarischer Symbole, 2. Aufl., Stuttgart 2012, S. 65.

12 Der Topos kommt bereits in den theologischen Schriften von Tertullian und Prosper, Leo dem Großen, Apringius, Basilius, Gregor von Nyssa und Gregor dem Großen vor. Eine besondere Stellung nimmt er in der Theologie von Johannes Chrysostomus sowie in den Werken des spanischen Juden Salomo Ibn Gabirol (11.Jh.), Herbert von Boshams und Luis“ von Granada ein. Vgl. Friedrich Ohly, Neue Zeugen des „Buchs der Natur“ aus dem Mittelalter, in: Hagen Keller, Nikolaus Staubach (Hrsg.), Iconologia sacra. Mythos, Bildkunst und Dichtung in der Religions- und Sozialgeschichte Alteuropas [Arbeiten zur Frühmittelalterforschung. Schriftenreihe des Instituts für Frühmittelalterforschung der Universität Münster, Bd. 23], Berlin, New York 1994, S. 546-568, Anm. 2, S. 546. Monika Schmitz-Emans nennt auch andere Kirchenväter - Hugo von Sankt Viktor, Raimund von Sabunde, Bonaventura, Alanus von Lille, denen die Natur ,als ein Buch, welches sich als Medium der Selbstoffenbarung Gottes komplementär zur Bibel verhält“ erscheint. Vgl. Monika Schmitz-Emans, Entzifferung, Buchstabieren und Konjektur. Aspekte und Funktionen des Weltschriftgleichnisses bei Georg Christoph Lichtenberg. In: „Jahrbuch der Lichtenberg-Gesellschaft“ 1991, S. 29-58, hier: S. 31.

13 Monika Schmitz-Emans, Buch, S. 65. In den naturphilosophischen Spekulationen, in denen die Zahlen und Buchstaben als Elemente des Systems gelten, steht der Topos auch mit dem hebräischen, Abraham zugeschriebenen „Buch der Schöpfung“(„Sepher Jezirah“), im Zusammenhang. Sie sind wahrscheinlich auf das 2 Jh. oder das 6 Jh. vor Christi zu datieren. Im 13. Jahrhundert wurde jene Zahlensymbolik und Zahlenkombinatorik von Raimundus Lullus aufgegriffen. Diese Konzeption stützte sich auf die Analogie zwischen physischen Elementen und religiösen Überzeugungen. Vgl. Friedrich Ohly, Neue Zeugen des „Buchs der Natur“, S. 552.

14 Gerhard Sauder, Aufklärerische Bibelkritik und Bibelrezeption, S. 109.

15 Mehr dazu: Yvonne Al-Taie, Tropus und Erkenntnis. Sprach- und Bildtheorie der deutschen Frühromantik, Göttingen 2015, S. 199-208. Francis Bacon schrieb vom Alphabet der Natur, Descartes forderte zum Lesen im Buch der Natur auf. Leibniz suchte nach einer Idealsprache, nach einem „Alphabet des Denkens“, das der Schrift in diesem Buch entsprechen würde. Vgl. Elisabeth Pernkopf, Alphabetisierte Natur, S. 81.

16 Gerhard Sauder, Aufklärerische Bibelkritik, S. 116. 
z.B. von Johann Georg Hamann, Johann Kaspar Lavater, Johann Gottfried Herder, Immanuel Kant, Johann Christoph Lichtenberg oder Johann W. von Goethe. ${ }^{17}$ Die Popularität des Topos lässt sich auch durch die Religionskritik und die Suche nach anderen Glaubensformen in der Aufklärung erklären. Der Topos wird offensichtlich zur Konkurrenz für die Bibel, deren Autorität durch die aufklärerische Offenbarungskritik geschwächt wurde. ${ }^{18}$

Einen Einfluss auf die Romantik hatte die Signaturenlehre von Paracelsus und später Jakob Böhme, deren Überzeugung von der „Sympathie von Zeichen und Bezeichnetem“, von der „Wechselwirkung von Name und Sache" und deren Reflexion über die Analogie zwischen Sprache und zeichenhafter Welt in der Literatur um 1800 erneut aufgegriffen wurde. ${ }^{19} \mathrm{Nicht}$ weniger bedeutend war, dass Böhme das Verstehen der Natur mit ihrer Unverständlichkeit in Zusammenhang brachte. Seine Lehre findet einen Widerklang in der Dichtung von Ludwig Kosegarten, Friedrich von Hardenberg (Novalis), Ludwig Tieck, Friedrich Schlegel oder - in satirischer Form - in den „Nachtwachen von Bonaventura“ von Klingemann.

\section{Zum Problem der Undarstellbarkeit des Buchs der Natur}

Mit der sprachlichen Darstellung des Transzendenten, das sich im Topos vom Buch der Natur widerspiegeln soll, geht seine paradoxe Unvorstellbarkeit einher: ${ }^{20}$ Einerseits versinnbildlicht er eine höhere Präsenz, die sich hinter der Natur verbirgt, andererseits kann er mit keinem konkreten Bild assoziiert werden, das das Undarstellbare ${ }^{21}$ aufzuzeigen vermöchte. Was sind die sichtbaren Träger dieser Offenbarung? In die Metapher des von Gott geschriebenen und chiffrierten Buches ${ }^{22}$ ist ein kognitiver (und subjektiver) Prozess eingeschlossen, an dem ein „Leser" beteiligt ist und von dem ihre Interpretation abhängt. Die ,absolute Metapher ${ }^{6}$ der Lesbarkeit der Welt ${ }^{23}$ ist mit anderen Tropen wie Schrift, Zeichen, Buchstaben, Zahlen, Chiffren, Hieroglyphen verbunden, die in der Geschichte ihre Rolle und Bedeutung ändern. Immer aktuell bleibt die Feststellung von Henri Veldhuis: „Wenn die Metapher des Buches für die Natur zutrifft, stellt sich die Frage, in welcher Sprache das Buch geschrieben ist?"24

17 Vgl. Monika Schmitz-Emans, Entzifferung, S. 29-58.

18 Ebd., S. 109. Infolge der Entdeckungen der Naturwissenschaften distanzierte sich auch die Philosophie von der Theologie.

19 Andreas Kilcher, Das ästhetische Sprachparadigma der Kabbala in der Romantik, in: ders., Die Sprachtheorie der Kabbala als ästhetisches Paradigma. Die Konstruktion einer ästhetischen Kabbala seit der Frühen Neuzeit, Stuttgart, Weimar 1998, S. 239-328, hier: S. 300.

20 Vgl. Ralf Simon, Die Bildlichkeit des lyrischen Textes. Studien zu Hölderlin, Brentano, Eichendorff, Heine, Mörike, George und Rilke, München 2011, S. 31.

21 Petra Bahr, Darstellung des Undarstellbaren. Religionstheoretische Studien zum Darstellungsbegriff bei A.G. Baumgarten und I. Kant, Tübingen 2004.

22 Ebd., vgl. auch: Friedrich Ohly, Neue Zeugen des „Buchs der Natur“, S. 546. Während die Heilige Schrift für das Ohr bestimmt war, richtete sich die Mitteilung Gottes durch die Natur an das Auge.

23 Hans Blumenberg, Die Lesbarkeit der Welt, 2. Aufl., Frankfurt a.M. 1983.

24 Henri Veldhuis, Ein versiegeltes Buch, S. 27. In der Anmerkung 41 (S. 27) findet man auch eine kurze Bibliographie zu diesem Thema. 
Obwohl sich der Topos säkularisiert hat, scheint sein religiöser Ursprung für die Interpretation seiner Rolle im Text relevant zu bleiben. Die Idee der Lesbarkeit der Welt geht nämlich auf die Johanneische Logoslehre zurück (Joh. 1, 1-3), nach der alle Geschöpfe aus dem göttlichen Logos hervorgingen. ${ }^{25}$ Aus Johannes' Logoslehre ergab sich der Dualismus zwischen dem schöpferischen Gotteswort und dem toten Buchstaben.

Dem von Ludwig Klages eingeführten Logozentrismus liegt auch die Überzeugung zugrunde, dass „eine absolute, außersprachliche Präsenz" vorhanden ist ${ }^{26}$ die die Bedeutung der sprachlichen Ausdrücke beeinflusst. In Hinsicht darauf scheint die Auseinandersetzung mit der Sprache als Mittel der Erkenntnis und mit ihrer metaphorischen Auffassung im Naturbuchkonzept begründet zu sein. ${ }^{27}$ Es wird aus räumlichen Gründen außer Acht gelassen, dass die beiden Topoi - die Sprache der Natur und das Buch der Natur - in der Forschung nicht immer gleichgesetzt werden. ${ }^{28}$ Es wird davon ausgegangen, dass die Natur „ein umfassendes nichtkonventionelles Zeichensystem“ ist und „einen Symbolzusammenhang darstellt, durch den sie sich all denjenigen offenbart, die dieses ,Buch der Natur zu lesen imstande sind.“29

\section{„Das Unsichtbare, das über uns schwebt, ziehen Worte nicht in unser Gemüt herab“}

Bereits in der literarisierten Physikotheologie von Barthold Hinrich Brockes („Irdisches Vergnügen in Gott", 1721-1748) wird die Metapher vom Buch der Natur einer literarisch-ästhetischen Modifizierung unterzogen, in der die Natur nicht nur gelesen, sondern auch neu geschrieben wird. ${ }^{30}$ Sie bleibe auch "grundsätzlich interpretierbar, ihre Buchstabenschrift kann lesen und begreifen, wer über die nötige Verstandeskraft und Einsicht verfugt". 31

Im ausgehenden 18. Jahrhundert lässt sich eine Metamorphose des Weltbuchkonzepts nachvollziehen: die optimistische Überzeugung von der „Lesbarkeit“ der Sprache der Natur wird durch die Erkenntnis- und Sprachkrise und den Kritizismus von Kant geschwächt. Die in geheimen Zeichen (Chiffren) oder Hieroglyphen sprechende Natur wird in zunehmendem Maße unverständlich. Während die Welt von Brockes als lesbares Buch aufgefasst wird, ${ }^{32}$

25 Vgl. Monika Schmitz-Emans, Entzifferung, S. 31-32.

26 Doris Feldmann, Hannah Jacobmeyer, Logozentrismus [Artikel], in: Ansgar Nünning (Hrsg.), Metzler Lexikon Literatur- und Kulturtheorie: Ansätze - Personen - Grundbegriffe, 3. Aufl., Stuttgart 2004, S. 406.

27 Vgl. Winfried Nöth, Ökosemiotik, in: ders., Handbuch der Semiotik, 2., vollständig neu bearbeitete und erweiterte Aufl., Stuttgart, Weimar 2000, S. 250-253, hier: S. 251.

28 Hans Blumenberg, Die Lesbarkeit der Welt, Anm. 200, S. 21.

${ }_{29}$ Geert Keil, Die naturalistische Herausforderung, in: ders., Kritik des Naturalismus, Berlin, New York 1993, S. 22-171, hier: S. 84.

30 Josef Billen, Friedhelm Hassel, Undeutbare Welt. Sinnsuche und Entfremdungserfahrung in deutschen Naturgedichten von Andreas Gryphius bis Friedrich Nietzsche, Würzburg 2005, S. 17-63, hier: S. 60.

31 Peter-André Alt, Aufklärung. Lehrbuch Germanistik, 2. Aufl., Stuttgart, Weimar 2001, S. 136.

32 Monika Schmitz-Emans, Buch, S. 65. 
wird sie in der Romantik für „eine grosse Gabe des Himmels“ gehalten, ${ }^{33}$ die „unzulänglich für die Erfassung des Wichtigsten" ist. ${ }^{34}$

Im Aufsatz „Von zwey wunderbaren Sprachen und deren geheimnißvoller Kraft“ aus den „Herzensergiessungen eines kunstliebenden Klosterbruders“ (1796) von Wilhelm Heinrich Wackenroder (1773-1798) und Ludwig Tieck (1773-1853) wird der transzendentale Charakter der Sprache von Natur und Kunst diskutiert und poetisch geschildert.

In den Vordergrund rückt die Ähnlichkeit zwischen den beiden metaphorisch aufgefassten Sprachen. Das Problem der Unmöglichkeit, das Transzendente richtig in Worte zu fassen, wird figurativ geschildert: „Nur das Unsichtbare, das über uns schwebt, ziehen Worte nicht in unser Gemüt herab“ (WHW 191), indem diese Unerreichbarkeit durch Verben und Präpositionen mit raumorientierter Bedeutung betont wird („schweben“, „über uns“, „herabziehen“), die auf eine religiös-hierarchische Ordnung im Verhältnis Gott-Mensch-Wortsprache verweisen: die Gottheit befindet sich oben, der Mensch unten, dazwischen „schweben“ Worte, die als Mittler zwischen zwei Wirklichkeitsdimensionen versagen. Diese Raumordnung bleibt auch im letzten Textfragment beibehalten, das als Antithese der vorherigen Formulierung denkbar ist: „die ewig lebendige, unendliche Natur, ziehet uns durch die weiten Räume der Lüfte unmittelbar zu der Gottheit hinauf" (WHW 193). In diesem Textabschnitt kommen philosophische („das Unsichtbare“), psychologische („Gemüt“) und sprachliche Begriffe nebeneinander vor, wodurch der Zusammenhang zwischen dem Heiligen, dem Inneren und der irrationalen Kommunikationsart aufgebaut wird. Darüber hinaus werden die mit dem Unendlichen verbundenen Konnotationen wiederholt und durch Epitheta verstärkt (für die „unendliche“, „lebendige“ Natur stehen „weite Räume der Lüfte“).

Yvonne Al-Taie zufolge bleibt die Sprachkrise, die am Anfang des Textes von Tieck und Wackenroder akzentuiert wurde, ausschließlich „auf den Bereich des Numinosen beschränkt. ${ }^{35}$ Es wird zwar weiter vom fiktiven Klosterbruder festgestellt: ,wir verstehen nur uns untereinander", aber der mystische Topos der „Sympathie des Menschen mit den Dingen “36 und der Menschen untereinander verstärkt nur die Ohnmacht der Sprache, die dem Inneren, dem menschlichen Gemüt gegenübergestellt wird: durch diese „wunderbare Sympathie“ werden "Gefühle oder Gesinnungen, oder wie man es nennen mag“ ins „Menschenherz“ zugeführt, „welche wir nie durch die abgemessensten Worte erlangen“ (WHW 192).

Trotz der Sprachkrise, die sich im Fragment anbahne, ${ }^{37}$ wird der Text in stilistischrhetorischer Hinsicht sorgfältig gepflegt. Yvonne Al-Taie zufolge benutzt der Klosterbruder „die geläufigen Topoi einer idyllischen Landschaftsbeschreibung“, eine „Vielzahl von rhe-

33 Wilhelm Heinrich Wackenroder, Werke und Briefe, Berlin und München 1984, S. 190-194, hier: S. 190 [Herzensergießungen eines kunstliebenden Klosterbruders: Von zwei wunderbaren Sprachen und deren geheimnisvoller Kraft], weiter als Sigle: WHW. Vgl. auch: Monika Schmitz-Emans, Romantische Sprachästhetik, in: Horst Albert Glaser, György Mihály Vajda (Hrsg.), Die Wende von der Aufklärung zur Romantik 1760-1820, S. 567-587, S. 573. Ähnliche Bemerkungen zur Unverständlichkeit der Wortsprache kann man auch im Werk von Georg Ch. Lichtenberg finden. Vgl. Monika Schmitz-Emans, Entzifferung, S. 29.

34 WHW 190. Vgl. auch: Monika Schmitz-Emans, Romantische Sprachästhetik, S. 573.

35 Yvonne Al-Taie, Tropus und Erkenntnis, S. 216.

36 Ebd., S. 218

37 Ebd., S. 216 
torischen Figuren“",38 dennoch wird die Wirkung „sinnlich-visueller Erlebnisqualitäten auf den Betrachter “ ${ }^{\text {39 }}$ durch die Anwendung von nahezu identischen Formulierungen erreicht. Paradoxerweise wird das Pathos des Sprechens (in) der Natur mit einer konventionellen Topik und Stilistik wiedergegeben. Diesen Kontrast verstärkt noch die Anspielung auf das biblische Motiv des toten Buchstabens, dem der fehlende Ton der „leeren Schallen“ ähnlich ist: „unser Ohr [wird] mit leeren Schallen gefüllt" (WHW 191).

Das Verstehen der transzendentalen Wirklichkeit, das kein rationaler, sondern ein auf Gefühle ausgerichteter Prozess ist, kann nach Wackenroder dank „zwei wunderbare[n] Sprachen" möglich sein:

Ich kenne aber zwei wunderbare Sprachen, durch welche der Schöpfer den Menschen vergönnt hat, die himmlischen Dinge in ganzer Macht [...] zu fassen [...]. Sie kommen durch ganz andere Wege zu unserm Inneren, als durch die Hülfe der Worte [...]. Die eine dieser wundervollen Sprachen redet nur Gott; die andere reden nur wenige Auserwählte unter den Menschen, die er zu seinen Lieblingen gesalbt hat. Ich meine: die Natur und die Kunst (WHW 191).

Die Sprache der Natur kommt hier in ihrem primären religiösen Kontext zum Vorschein: dieses „gründlichste und deutlichste Erklärungsbuch“ über das ,Wesen` Gottes „und seine Eigenschaften" wird mit der Kunst verglichen, die ähnlich wie andere Naturerscheinungen „ins Innere" trifft. ${ }^{40}$ Die beiden Sprachen berühren „den Menschen als Ganzen“, sie umfassen seine psycho-somatische Einheit, ${ }^{41}$ die dem Gedanken von der kosmisch-sympathetischen Einheit entspricht.

Die Sprache der Natur wird im Aufsatz, kunstvoll und konventionell zugleich, mithilfe von idyllischen Naturerscheinungen exemplifiziert:

Das Säuseln in den Wipfeln des Waldes, und das Rollen des Donners, haben mir geheimnisvolle Dinge von ihm erzählet, die ich in Worten nicht aufsetzen kann. Ein schönes Tal, von abenteuerlichen Felsengestalten umschlossen, oder ein glatter Fluß, worin gebeugte Bäume sich spiegeln, oder eine heitere grüne Wiese von dem blauen Himmel beschienen, - ach diese Dinge haben in meinem inneren Gemüte mehrwunderbare Regungen zuwege gebracht, haben meinen Geist von der Allmacht und Allgüte Gottes inniger erfüllt, und meine ganze Seele weit mehr gereinigt und erhoben, als es je die Sprache der Worte vermag (WHW 191).

Um die Vielfalt der Naturerscheinungen wiederzugeben, nimmt Wackenroder hier die syntaktische Figur der accumulatio in Anspruch. Das Polysyndeton, eine vielfache Verbindung von Wörtern durch die gleichen Konjunktionen ,und' beziehungsweise ,oder', liegt hier neben einer Antithese („das Säuseln [...] des Waldes“versus „das Rollen des Donners“) vor, deren Semantik auf die alttestamentliche Darstellung Gottes anspielt, der sich in den Gegensätzen offenbart (vgl. 1 Könige 19,11-12).

38 Ebd., S. 217

39 Ebd., S. 218. Außer dieser Feststellung wird in der Arbeit von Yvonne Al-Taie dem Stil des Textes keine Aufmerksamkeit geschenkt. In den anderen Beiträgen zur Schrift von Wackenroder und Tieck findet man auch keine eingehendere Analyse von rhetorischen Tropen im Aufsatz. Vgl. dazu: Dirk Kemper, Sprache der Natur, in: ders., Sprache der Dichtung. Wilhelm Heinrich Wackenroder im Kontext der Spätaufklärung, Stuttgart 1993, S. 172-181.

40 Monika Schmitz-Emans, Romantische Sprachästhetik, S. 573.

41 Yvonne Al-Taie, Tropus und Erkenntnis, S. 216 
Der Sprache der Natur wird die Unmöglichkeit gegenübergestellt, sie zu beschreiben (,in Worten nicht aufsetzen“, „als es je die Sprache der Worte vermag“) oder zu verstehen oder mit den „Dingen“ zu kommunizieren:

[...] eine unendliche Menge von Dingen [...], wovon jedes ein anderes Wesen hat, und wovon wir keines verstehen und begreifen. Wir wissen nicht, was ein Baum ist; nicht, was eine Wiese, nicht, was ein Felsen ist; wir können nicht in unsrer Sprache mit ihnen reden; wir verstehen nur uns untereinander. Und dennoch hat der Schöpfer in das Menschenherz eine solche wunderbare Sympathie zu diesen Dingen gelegt, daß sie demselben, auf unbekannten Wegen, Gefühle oder Gesinnungen, oder wie man es nennen mag, zuführen, welche wir nie durch die abgemessensten Worte erlangen (WHW 192).

Die Satzkonstruktionen mit dem Asyndeton (in dem Aufzählungen durch Kommata getrennt werden) und Polysyndeton (mit der Konjunktion ,und') sowie die Anhäufung von Verneinungen (,nicht' ${ }^{\prime}$ zeigen das Ausmaß an Unwissen. Trotz der Mangelhaftigkeit der Wortsprache kommt die Kommunikation der Gottheit mit den Menschen zustande, in der Gott als Autor bzw. Künstler dargestellt wird. Auch dabei greifen Wackenroder und Tieck einen Topos auf, der in den irrationalen Schriften der Aufklärung verbreitet war.

\section{Gott als Autor und Künstler}

Im Topos vom Buch der Natur kommuniziert dessen Autor (der Geist) mit seinen Lesern (Menschheit, im Text von Wackenroder und Tieck als, wir' genannt). In der Romantik wird das Buch der Natur ästhetisiert, indem eine „Vorstellung von Gott als dem Poeten der Welt ““22, die wiederum für die „,vollkommene Schrift aus der Autorenschaft Gottes ${ }^{“ 43}$ gehalten wird, in den Vordergrund rückt. Johann Georg Hamann weist auf dieses Verhältnis in einem Brief an Friedrich Heinrich Jacobi aus dem Jahr 1784 hin:

Gott, Natur und Vernunft haben eine so innige Beziehung auf einander, wie Licht, Auge und alles, was jenes diesem offenbaret, oder wie Mittelpunkt, Radius und Peripherie jedes gegebenen Cirkels, oder wie A u t o r, Bu ch und Le s e r. Wo liegt aber das Räthsel des Buchs? In seiner Sprache oder in seinem Inhalt? Im Plan des Urhebers oder im Geist des Auslegers $?^{44}$ [hervorgeh. von AKH].

Im zitierten Textfragment wird zwischen der Sprache der Natur und dem Inhalt ihres Buches differenziert, wobei die Antwort auf die eigene Frage offen gelassen wird. Auch in Hamanns Aussage kommen Polysyndeta vor: „Gott, Natur und Vernunft“, „Mittelpunkt, Radius und Peripherie“, „Autor, Buch und Leser“, die eine Verbundenheit der erwähnten Elemente hervorhebt.

Der Klosterbruder Wackenroders stellt Gott nicht als Autor, sondern als Künstler dar, was mit der Fragwürdigkeit der Wortsprache in der Wahrheitsvermittlung zusammenhängt:

42 Petra Bahr, Darstellung des Undarstellbaren, S. 198.

43 Ebd.

44 Johann Georg Hamann's Schriften und Briefe in vier Theilen. Vierter Theil, Hannover 1874, S. 208. 
Die Natur, soviel davon ein sterbliches Auge sieht, gleichet abgebrochenen Orakelsprüchen aus dem Munde der Gottheit. Ist es aber erlaubt, also von dergleichen Dingen zu reden, so möchte man vielleicht sagen, daß Gott wohl die ganze Natur oder die ganze Welt auf ähnliche Art, wie wir ein Kunstwerk, ansehen möge (WHW 194).

Der bisher christlich-religiöse Argumentationsvorgang weicht einer Anspielung auf die Orakel in Delphi, wodurch ein typisch romantischer Zusammenhang zwischen Religionen, zwischen der griechischen Mythologie und dem Christentum, aufgebaut wird. Paradoxerweise gehört der Orakelspruch zur Wortsprache und zu derselben Rätsel-Metaphorik wie die Chiffren- oder Hieroglyphenmetapher - die Interpretation der Mitteilung wird dem Menschen überlassen, das Rätselhafte „aus dem Munde der Gottheit“ (WHW 194) wird ästhetisiert, in Kunst verwandelt, deren Bildhaftigkeit dem sprachlichen Ausdruck weicht (Metonymie „Orakelsprüche[]" sowie Verben wie „reden“, „sagen“ verweisen auf den mündlichen Charakter der göttlichen Sprache, die dem toten Buchstaben gegenübersteht (?)).

Hamann und die Romantiker trennt eine Generationenkluft, aber sowohl der ,Magus des Nordens', der in seiner Schrift auf die Mathematik und ihre Regeln anspielt, als auch die Autoren des poetischen Essays versuchen, die Kommunikation zwischen dem Göttlichen und dem Menschen greifbar zu machen. Die Zusammenfügungen und die accumulatio dienen der Hervorhebung dieser Zusammenhänge.

Da die Sprache der Natur aus dem Übernatürlichen hervorgeht, das nicht verstanden wird, verweist das Buch der Natur auf die problematische „Relation zwischen Sprache und Transzendenz“, in der „die Grenzen der Sprache nachdrücklich erfahren“ werden. ${ }^{45}$ Diese Grenzen werden jedoch mithilfe von rhetorischen Figuren aufgehoben, die als sprachliche Widerspiegelung einer geheimnisvollen Verbindung zwischen den Dingen, als „Sympathie“ gelten können.

\section{Einheit in der Vielfalt}

Einen ähnlichen Satzbau in der Beschreibung der "Chiffernschrift“ trifft man im naturphilosophischen Roman-Fragment „Lehrlinge zu Sais“von Novalis an, das zwischen den Jahren 1798 und 1799 verfasst und posthum 1802 von Schlegel und Tieck veröffentlicht wurde.

Unter Chiffre wird eine Geheimschrift verstanden, „in der die Buchstaben nach einem verschlüsselten System (Code) durch andere Zeichen (Buchstaben, Ziffern) ersetzt werden “ ${ }^{46}$ In der Philosophie und Metaphysik ist die Chiffre „Ausdruck für den Bedeutungsträger einer höheren Erkenntnis und Einsicht “. ${ }^{77}$ Die Chiffre als verschlüsselte Rede (und rhetorische Figur par excellence!) gewinnt neben der Hieroglyphenmetapher bereits in der Rhetorik und Emblematik des Barock an Bedeutung. Im 18. Jahrhundert wird sie ,zu einer besonderen Form

45 Monika Schmitz-Emans, Romantische Sprachästhetik, S. 571-572.

46 Isabel Zollna, Chiffre [Artikel], in: Gert Ueding, Gregor Kalivoda u.a. (Hrsg.), Historisches Wörterbuch der Rhetorik, Bd. 2, Tübingen 1994, S. 173-174, hier: S. 173.

47 Ebd. 
des änigmatischen (rätselhaften) und manieristischen Ausdrucks “ ${ }^{48}$ Die Chiffre als rhetorische Figur gilt innerhalb der elocutio als Beispiel „der ,Dunkelheit' des sprachlichen Ausdrucks“ .49

Novalis, dem die Idee der Weltsympathie und Böhmes Signaturenlehre besonders nah waren, verwendet den Begriff „Chiffernschrift“ ${ }^{\text {“50 }}$ nicht ausschließlich in Bezug auf die Natur, sondern auf „menschliche Wege“. Die „Chiffernschrift“ gewinnt eine geschichtliche Dimension, indem sie auf räumlicher wie zeitlicher Ebene, im Mikro- und Makrokosmos vorhanden ist. Damit wird das Buch der Natur mit dem Buch der Geschichte konfrontiert:

Wer sie [menschliche Wege] verfolgt und vergleicht, wird wunderliche Figuren entstehen sehn; Figuren, die zu jener großen Chiffernschrift zu gehören scheinen, die man überall, auf Flügeln, Eierschalen, in Wolken, im Schnee, in Krystallen und in Steinbildungen, auf gefrierenden Wassern, im Innern und Äußern der Gebirge, der Pflanzen, der Thiere, der Menschen, in den Lichtern des Himmels, auf berührten und gestrichenen Scheiben von Pech und Glas, in den Feilspänen um den Magnet her, undsonderbaren Conjuncturen des Zufalls, erblickt. In ihnen ahndet man den Schlüssel dieser Wunderschrift, die Sprachlehre derselben (NL 95) [hervorgeh. von AKH].

Bei Novalis bezieht sich die „Chiffernschrift" nicht nur auf die Natur, sondern auch im psychologischen Sinne auf alle Erscheinungen, die sich in den menschlichen Tätigkeiten beobachten lassen. Damit wird das Bewusstsein in die Vision des Weltbuches eingeschlossen. Durch die Einführung dieser polysyndetischen (unverbundenen) Aufzählung konstruiert der Dichter ein entsprechendes synthetisches Bild des Ganzen, in dem alle Elemente getrennt gesehen (und genannt!) werden und zugleich eine Ganzheit bilden. Wenn man den Einfluss des subjektiven Idealismus von Fichte berücksichtigt, kann man vermuten, dass diese angestrebte Ganzheit nur im Ich erreichbar ist. Trotzdem „ahndet“ man nur „,den Schlüssel dieser Wunderschrift“, die nicht eindeutig und verständlich ist.

Der Erkenntnisweg und die zu beobachtenden Phänomene werden mithilfe ähnlicher rhetorischer Mittel gestaltet, vor allem der Aufzählung (enumeratio). Satzkonstruktionen wie „verfolgt und vergleicht“, „auf berührten und gestrichenen Scheiben“, „Scheiben von Pech und Glas" sind syndetisch aufgebaut.

Mithilfe der gleichen rhetorischen Mittel stellt das Buch der Natur Johannes Chrysostomus (gest. 407), ,einer der größten Prediger des Lobes Gottes aus der Schöpfung " dar, ${ }^{51}$ der viele Gedanken der Physikotheologen des 18. Jahrhunderts vorgebildet hat. ${ }^{52}$

Das asyndetische Aufzählen von mindestens drei Wörtern oder Satzgliedern, die gleichgestellt sind, dient dem mittelalterlichen Theologen dazu, die von der Erde hervorgebrachten Güter - „Samen, Kräuter[] und Pflanzen, Blumen, Wiesen, Gärten“ [hervorgeh. von $\mathrm{AKH}]^{53}$ in ihrer Mannigfaltigkeit darzustellen.

48 Ebd.

49 Ebd., S. 174.

50 Novalis, Die Lehrlinge zu Sais, in: ders., Werke, herausgegeben und kommentiert von Gerhard Schulz, München 1969, S. 95. Weiter als Sigle NL.

51 Friedrich Ohly, Neue Zeugen des „Buchs der Natur“, S. 546.

52 Ebd.

53 Ebd., S. 547. Falls nicht anders angegeben, wurden die Zitate aus den Schriften von Johannes Chrisostomus dem Beitrag von F. Ohly entnommen. 
Johannes Chrysostomus' Naturauffassung ist noch aus einem anderen Grund wichtig: Er reflektiert über den rätselhaften Charakter ihrer Sprache. Er lässt „die Himmel“ den Ruhm Gottes sprachlos verkünden - „ohne Stimme, Mund und Sprache, allein durch ihren Anblick“ [hervorgeh. von AKH]. ${ }^{54}$ „Der Himmel schweigt [...], denn dieser Sinn [Augen] ist von Natur her zuverlässiger und sicherer als jener" [hervorgeh. von AKH]..$^{55}$

Das Schweigen, die Wortlosigkeit der Natur, schließen jedoch ihre Kommunikation mit den Menschen durch Bilder und Zeichen nicht aus. Einerseits rät Chrysostomus dazu, von der Natur zu sprechen: „Laßt uns sprechen von der Erde [...], dem Mannigfaltigen und Verschiedenen, das sie hervorbringt, den Samen, Kräutern und Pflanzen, Blumen, Wiesen, Gärten" [hervorgeh. von AKH], ${ }^{56}$ andererseits sieht er in den Dingen „die unaussprechliche Philosophie“: „Mit diesen Dingen also laßt uns umgehen, und von dort soll uns das größte Vergnügen kommen, von dort Gewinn, von dort die unaussprechliche Philosophie“ [hervorgeh. von AKH]. ${ }^{57}$

Bei der Beschreibung der Natursprache nutzt dieser Kirchenlehrer des Ostens die rhetorischen Sprachmittel der Worthäufung (accumulatio). Zu diesen gehört das Hendiadyoin, das einen Vorgang durch zwei sich ergänzende Begriffe ausdrückt, wobei aus drei Wörtern ein anderer Begriff entsteht, der eine neue Bedeutung hat („Stimme, Mund und Sprache"), das Asyndeton, eine Aufzählung aus mindestens drei Teilen, die ohne Konjunktionen miteinander verbunden sind („Pflanzen, Blumen, Wiesen, Gärten“) oder die Anapher (,und von dort [...], von dort [...]").

Eine ähnliche Satzkonstruktion findet man bei Hamann, der das Buch der Natur aus der Heiligen Schrift heraus interpretiert. In „Brocken“ scheint er die Wahrheit der Mitteilung von der Natur sogar in Frage zu stellen:

Alle Erscheinungen der Natur sind Träume, Gesichte, Rätsel, die ihre Bedeutung, ihren geheimen Sinn haben. Das Buch der Natur und Geschichte sind nichts als Chyffren, verborgene Zeichen, die eben den Schlüssel nötig haben, den die Heilige Schrift auslegt und die Absicht ihrer Eingebung ist ${ }^{58}$ [hervorgeh. von $\mathrm{AKH}$ ].

Der Asyndeton „Träume, Gesichte, Rätsel“verweist auf die Ganzheit der Naturbetrachtung, aber auch auf eine erahnte Subjektivität der Wahrnehmung. Eine Kontinuität dieses Gedankens findet man bei Novalis, der die Weltbetrachtung ins Bewusstsein zu übertragen versuchte.

54 Friedrich Ohly, Neue Zeugen des „Buchs der Natur“, S. 548.

55 Ebd., S. 548. Es wird hier außer Acht gelassen, wie sich die deutsche Übersetzung zum Original verhält. In einer anderen Übersetzung kann man beispielsweise lesen: „Die Himmeln erzählen die Herrlichkeit Gottes. Sage mir, wie erzählen sie denn diese? Sie haben keine Stimme, sie haben keine Lippen, sie haben keine Zunge; wie erzählen sie denn diese? Durch den Anblick selbst." (Des heiligen Kirchenlehrers Johannes Chrysostomus Predigten und kleine Schriften, Bd. 3, Prag 1785, S. 216). Es ist deutlich, dass die Satzkonstruktion geändert wurde, aber die Häufung wurde beibehalten.

56 Friedrich Ohly, Neue Zeugen des „Buchs der Natur“, S. 547 (In ps.9.2., PG 55, 123).

57 Ebd., S. 548 (In ps.9,3, PG 55, 124).

58 J.G. Hamann, Brocken, in: ders., Sämtliche Werke. 6 Bände, hrsg. von J. Nadler, Bd. 1, Wien 1949-1957, S. 308. 


\section{(Toter) Buchstabe und (heilige) Hieroglyphe}

Das literarische und philosophische Werk von Novalis ist durch die sprachmystische Thematik gekennzeichnet, die wiederum auf die Rezeption der Mystik von Böhme zurückgeht. Der Dichter versteht die Sprache der Natur als Mitteilung des Geistes, die nicht mehr verstanden wird. Die paulinische Opposition von Geist und Buchstabe (vgl. II Kor, 3,6) liegt hier auf der Hand:

Alles, was wir erfahren, ist eine Mittheilung: so ist die Welt in der That eine Mittheilung, Offenbarung des Geistes. Die Zeit ist nicht mehr, wo der Geist Gottes verständlich war, der Sinn der Welt ist verloren gegangen, wir sind beim Buchstaben stehen geblieben [...] - Ehemals war alles Geisterscheinung, jetzt sehen wir nichts als todte Wiederholung, die wir nicht verstehn. Die Bedeutung der Hieroglyphe fehlt. Wir leben noch von der Frucht besserer Zeiten ${ }^{59}$ [hervorgeh. von AKH].

Novalis zufolge offenbart sich der Geist im chiffrierten oder hieroglyphischen Zeichensystem. Von der Sprache der Natur als „Medium der Offenbarung zwischen Gott [...] und den Menschen ${ }^{\text {“60 }}$ sind nur Buchstaben übrig geblieben. In der Topik gilt der Buchstabe als „Symbol des Äußerlichen, Materiellen und Toten“, ${ }^{61}$ des Partikulären, das erst im Prozess der Zusammenfügung, als Teil einer Ganzheit, seinen Sinn bekommt. In der paulinischen Tradition wird der tote Buchstabe dem lebendigen Logos, Christus, gegenübergestellt. Der Dualismus von sinnlich wahrnehmbarer Welt (mundus sensibilis), die Novalis für eine nicht verstandene „Mittheilung" hält, und geistig erkennbarer Welt (mundus intelligibilis), ${ }^{62}$ die im Goldenen Zeitalter als „Geistererscheinung“ empfunden wurde und in der Gegenwart als „todte Wiederholung" angesehen wird, wird jedoch im darauffolgenden Satz aufgehoben: „Wir leben noch von der Frucht besserer Zeiten“. ${ }^{63}$ Der nicht klar formulierte Satz verweist auf eine verborgene Anwesenheit des Göttlichen, die in die Gegenwart hinein - sei es als Erinnerung oder als Zukunftsvision - fortwirkt.

Die „todte Wiederholung“ wird dem Konzept der Wiederherstellung des Goldenen Zeitalters, der „besseren Zeiten“ entgegengesetzt. Novalis verbindet hier verschiedene Bedeutungen der Buchstabensymbolik. Der Buchstabe ist „Symbol des Rätselhaften“, ${ }^{64}$ aber der „tote Buchstabe" steht auch für oberflächliche Erkenntnis, die der intuitiven Erkenntnis aus dem Geiste widerspricht. Der einzelne Buchstabe verweist auf den unvollendeten hermeneutischen Prozess, in dem das aus den Buchstaben bestehende Ganze entsteht. Die Formulierung „,beim Buchstaben stehen bleiben" kann eine Auslegung der Welt bedeuten, die durch schemenhaftes Denken gehemmt ist, das eine Konzentration auf Einzelheiten bedeutet, während der das

59 Novalis, Vorarbeiten zu verschiedenen Fragmentsammlungen (1798), in: ders., Werke. Tagebücher und Briefe Friedrich von Hardenbergs, hrsg. von H.-J. Mähl, R. Samuel, Bd. 2, München 1978, S. 383.

60 Aleida Assmann, Schriftspekulationen und Sprachutopien in Antike und früher Neuzeit, in: Eveline Goodman-Thau, Gert Mattenklott, Christoph Schulte (Hrsg.), Kabbala und Romantik. Die jüdische Mystik in der romantischen Geistesgeschichte, Tübingen 1994, S. 23-41, hier: S. 23.

61 Monika Schmitz-Emans, Buchstabe, S. 67.

62 Vgl. ebd., S. 67.

63 Novalis, Vorarbeiten zu verschiedenen Fragmentsammlungen, S. 383.

64 Vgl. Monika Schmitz-Emans, Buchstabe, S. 68. 
Gesamtbild verloren geht. Es liegt auf der Hand, dass es sich nicht um ein rationales, sondern ein spirituelles Gesamtbild handelt. Dazu dient ein metonymischer Verweis auf die in Bildern verschlüsselte Wahrheit.

Als Novalis seinen Text schrieb, war die hieroglyphische Schrift noch nicht entziffert. Wegen der Komplexität des Schriftsystems und der falschen Annahme, dass es sich bei den Hieroglyphen um eine Bilderschrift handle, war das romantische Verständnis der Hieroglyphe von dem eines Champollion durchaus verschieden. ${ }^{65}$ In der Topik stehen die Hieroglyphen für Symbole von Phänomenen mit verschlüsselter Bedeutung. Im ausgehenden 18. Jahrhundert wurde der Begriff zu einem zentralen Schlagwort. Das Lehnwort (griech. hieros - heilig, priesterlich, glyphe - Schnitzwerk, Skulptur) bedeutete „ein in hartes Material (Stein) eingeschnittenes bzw. eingemeißeltes Zeichen einer Priesterschrift“ ${ }^{66}{ }^{6}$ "Heilig (hieros) seien jene Zeichen, die man in Stein eingeritzt (glyphein), als in der Natur, vorfindet" ${ }^{\text {" }}{ }^{67}$

Die Entzifferung von Hieroglyphen begann 1799 mit der Entdeckung des Steines von Rosette im westlichen Nildelta, in der Stadt Rosette (Rashid), auf dem drei Texte eingraviert waren, in Hieroglyphen sowie in demotischer und griechischer Übersetzung. Erst 1822 veröffentlichte Jean-François Champollion die Ergebnisse seiner Forschung und Entzifferung von Hieroglyphen. ${ }^{68}$

$\mathrm{Zu}$ Lebzeiten von Novalis gelten die Hieroglyphen als Chiffren mit bildlichem Charakter. Die Entstehung der Hieroglyphen als Metapher geht auf Hermes Trismegistos zurück. ${ }^{69}$ Bereits in der Antike werden sie mit einer mystisch-sakralen Dimension assoziiert. ${ }^{70}$ In der lateinischen Spätantike wird die Hieroglyphe als Symbolschrift verstanden, in der Frühen Neuzeit - als Bildersymbol. ${ }^{71}$ Nach Giambattista Vico, „Scienza Nuova Seconda“ (1744), war die erste Sprache hieroglyphisch. ${ }^{72}$ In der Romantik erscheint der Begriff Hieroglyphe „in Verbindung mit dem Unendlichen und Unfassbaren." ${ }^{\text {"73 }}$

In ähnlicher Bedeutung kommen Hieroglyphen auch in den Schriften von Shaftesbury, Kant, Herder, Lavater, Schiller und Humboldt ${ }^{74}$ vor. Die Hieroglyphen der Natur stellen eine übersinnliche Wahrheit dar, die in der Welt immanent ist und deren Aufschlüsselung nicht rational erfolgen kann. „[D]as ganze Leben und Weben der Natur [galt] als heilig [...]

65 Alexander Kupfer, Die künstlichen Paradiese: Rausch und Realität seit der Romantik. Ein Handbuch. Sonderausgabe, Stuttgart, Weimar 2006.

66 Klaus Weimar u.a. (Hrsg.), Reallexikon der deutschen Literaturwissenschaft: Neubearbeitung des Reallexikons der deutschen Literaturgeschichte, Bd. 1 A-G, Berlin, New York 2007, S. 46.

67 Alexander Kupfer, Die Hieroglyphensprache der Natur, in: ders., Die künstlichen Paradiese, S. 162-168, hier: S. 162.

68 Michael Höveler-Müller, Hieroglyphen lesen und schreiben. In 24 einfachen Schritten, München 2014, S. 23-29.

69 Erik Hornung, „Hieroglyphisch denken“, Bild und Schrift im alten Ägypten in: Gottfried Boehm, Stephan E. Hauser (Hrsg.), Homo pictor, München, Leipzig 2001, S. 76-86, hier: S. 84.

70 Almut-Barbara Renger, Hieroglyphe [Artikel], in: Günter Butzer, Joachim Jacob (Hrsg.), Metzler Lexikon literarischer Symbole, 2. Aufl., Stuttgart 2012, S. 181-182, hier: S. 181.

71 Ebd., S. 181.

72 Erik Hornung, „Hieroglyphisch denken“, S. 85.

73 Almut-Barbara Renger, Hieroglyphe, S. 181.

74 Monika Schmitz-Emans, Buchstabe, S. 68. 
und [ließ] einen verborgenen göttlichen Sinn ahnen “" ${ }^{75}$ Da man Hieroglyphen Anfang des 18. Jahrhunderts für aus Bildern abgeleitete Schriften hielt, wird das Bildhafte, das sich als Ganzheit sehen lässt, von Novalis dem Buchstaben eindeutig entgegengesetzt.

Das Verstehen der Welt als Hieroglyphe bedeutet für Novalis, sie zu übersetzen. Der Welterkenntnis wird dabei ein sprachlicher Charakter verliehen. Bei Novalis, ähnlich wie bei Hamann, der in jeder sprachlichen Tätigkeit eine Art Übersetzung aus einer göttlichen Ursprache sieht, ${ }^{76}$ besteht die Erkenntnis in der intuitiven oder mystischen Entzifferung der Hieroglyphe, die ein chiffriertes Bild darstellt.

Im zitierten Fragment modifiziert er jedoch die Bedeutung des Topos, indem er die Gegenüberstellung zwischen Offenbarung und Buchstaben, zwischen Transzendenz und Immanenz aufhebt. Das Transzendente bleibt nämlich durch seine Zeichen in der Welt immanent, die Wiederholung der alten spirituellen Ordnung ist als unverständliche Hieroglyphe vorhanden. Auch das Leben „von der Frucht besserer Zeiten“ schließt diese Transzendenz nicht vollständig aus. Das Problem liegt in der Unmöglichkeit, die wahrnehmbare Welt richtig auszulegen.

\section{Das Sammeln und Vergleichen: Signaturenlehre und ihr sprachlicher Ausdruck}

Für die romantische Rezeption des Topos ist seine Vorgeschichte relevant, die auf das neuplatonische Mikrokosmos-Makrokosmos-Schema zurückgeht. Die Auffassung des Kosmos als ein allgemeiner Sinnzusammenhang spiegelte sich in den naturmagischen Visionen des Ganzen sowie in ihrem irrationalen Konzept der verlorenen Ursprache wider. Insbesondere die Überzeugung von der Harmonie und den im Universum herrschenden Weltsympathien wird durch rhetorische Figuren aufgezeigt, die in der Literatur im Kontext des Weltschriftkonzepts angewendet wurden.

In „Lehrlinge zu Sais“ wird der geheimnisvolle Lehrer und sein Weg der Erkenntnis durch das Sammeln von Dingen gekennzeichnet, die für bestimmte Wissensbereiche stehen. Sie werden von dem Ich-Erzähler aufgezählt: „Er sammelte sich Steine, Blumen, Käfer aller Art, und legte sie auf mannichfache Weise sich in Reihen" (NL 96). Novalis wählt Motive aus dem Bereich des Anorganischen, der organischen Natur, in makro- und mikrokosmischer Dimension. Auf diese Weise entsteht eine nachgeahmte „Chiffernschrift“, die „des Weltalls Symphonie“ entspricht (NL 95). Sie nimmt eine synthetisierende Form an und verbindet "die empirischen Vereinzelungen der Dinge“ ${ }^{77}$ Diese Nachahmung der "Chiffernschrift" kann man im Stil von Novalis nachvollziehen. Wir haben es hier zugleich mit einer ähnlichen rhetorischen Figur wie bei den anderen Autoren zu tun - mit der enumeratio.

75 Ebd., S. 162.

76 Johann Georg Hamann, Aesthetica in nuce, in: Sämtliche Werke, Bd. 2, Wien 1950, S. 193-217, hier: S. 199.

77 Reinhard Leusing, Die Stimme als Erkenntnisform - zu Novalis 'Roman „Die Lehrlinge zu Sais“, Stuttgart 1993, S. 97. 
Das Sammeln von Wissen bezieht sich auch auf Erfahrungen und Betätigungen, die asyndetisch aufgezählt werden, die zusätzlich die auf die Fremdheit hinweisenden, synonymischen Epitheta („andere“, „neue“, „fremde“, „unbekannte“) betonen:

Wie er größer ward, strich er umher, besah sich andre Länder, andre Meere, neue Lüfte, fremde Sterne, unbekannte Pflanzen, Thiere, Menschen, stieg in Höhlen, sah wie in Bänken und in bunten Schichten der Erde Bau vollführt war, und drückte Thon in sonderbare Felsenbilder (NL 96) [hervorgeh. von $\mathrm{AKH}]$.

Mit dem Sammeln und Vergleichen als Vorgehensweise des Wissenserlangens geht die Erkenntnis der bestehenden Zusammenhänge einher:

Nun fand er überall Bekanntes wieder, nur wunderlich gemischt, gepaart, und also ordneten sich selbst in ihm oft seltsame Dinge. Er merkte bald auf die Verbindungen in allem, auf Begegnungen, Zusammentreffungen (NL 96) [hervorgeh. von AKH].

Reinhard Leusing bemerkt, dass die „anthropologischen und sozialen Verfassungen der Menschen [...] sich bruchlos in diese Universalität alles Beobachtbaren " reihen. ${ }^{78}$

Darüber hinaus werden bei der Erkenntnis alle Sinne und der Verstand im Textfragment berücksichtigt und asyndetisch bzw. syndetisch nebeneinander gereiht: „[...] er hörte, sah, tastete und dachte zugleich" (NL 96).

Andreas Kilcher zufolge stellt das Romanfragment die magische Sympathie dar. Einer „Korrespondenz zwischen Mikro- und Makrokosmos“, die hier auf der Hand liegt, entspricht in der Transzendentalphilosophie eine Korrespondenz zwischen Subjekt und Objekt. ${ }^{79}$ Die Synthese, das Lesen im Buch der Natur, wird auch auf der formal-ästhetischen Textebene erreicht.

Kilcher hebt die Einflüsse der hermetischen Schriften auf Novalis hervor. Den Namen Paracelsus hat sich Novalis nach der Lektüre von Dietrich Tiedemanns „Geist der spekulativen Philosophie“ sowie des Buches „Versuch einer pragmatischen Geschichte der Arzneikunde“ von Kurt Sprengel notiert. ${ }^{80}$ In den beiden Werken wird unter anderem die „Lehre der Signaturen" dargestellt, die von Novalis als Sprachmagie aufgefasst wird.

Darüber hinaus weist Kilcher auf den Zusammenhang der Weltauffassung von Novalis mit seiner Sprache hin: „Wie der Kosmos eine Harmonie korrespondierender Signaturen ist, so sind es auf der Ebene der poetischen Sprache die Bilder, Tropen und Begriffe" ${ }^{81}$ Die von Novalis gebrauchte Kombinatorik sei „nicht nur das Struktur- und Stilprinzip eines enzyklopädischen Wissens, sondern auch ein erfindendes, produktives, magisches Verfahren “. 82 Auf die Feinheiten des Sprachstils von Novalis geht Kilcher in seiner umfassenden Studie nicht ein. Es ist angebracht, ein Beispiel anzuführen.

78 Ebd., S. 97.

79 Vgl. Andreas Kilcher, Das ästhetische Sprachparadigma der Kabbala, S. 297.

80 Vgl. ebd., S. 277.

81 Ebd., S. 300.

82 Ebd. 
In Sprengels Versuch einer pragmatischen Geschichte der Arzneikunde wird die Signaturenlehre von Paracelsus folgendermaßen dargestellt: „Jedes Kraut, sagt er [Paracelsus], ist ein Stern, und jeder Stern ist ein Kraut" ${ }^{83}$ Die Lehre von Paracelsus wird jedoch von Sprengel auch einer Kritik unterzogen und "Torheit" genannt: „die Sterne sind Menschen wie wir" 84

Novalis verwandelt die Paracelsische Vision von der Natureinheit in eine sprachliche, indem er die Wechselwirkung von Naturdingen, Pflanzen und Tieren durch eine Anadiplose wiedergibt: ${ }^{85}$

Bald waren ihm die Sterne Menschen, bald die Menschen Sterne, die Steine Thiere ${ }^{86}$, die Wolken Pflanzen, er spielte mit den Kräften und Erscheinungen, er wußte wo und wie er dies und jenes finden, und erscheinen lassen konnte (NL).

Der rhetorischen Figur geminatio entspricht „Wechselwirkung der Signaturen der Dinge“. ${ }^{87}$

\section{Fazit}

Die göttliche Schrift im Buch der Natur verweist auf die Figur des Poeten, der als Eingeweihter den Schlüssel ihrer Interpretation hat. Darauf beziehen sich zum Beispiel die Frühromantiker Wackenroder und Novalis, aber auch Jean Paul in seiner „Vorschule der Ästhetik“ oder E.T.A. Hoffmann, der in seinem Werk auf das Hieroglyphenmotiv eingeht. Die ersten Spuren der Sprachkrise, die sich in der Epoche beobachten lassen, etwa im „Monolog “ von Novalis, haben sich auf das Verständnis der Kommunikation und des Verstehens ausgewirkt.

Der Topos, der im Mittelalter auf die Offenbarung Gottes in der Schöpfung verwies, korrespondierte mit dem Konzept der sprachhaften Natur, die in der Romantik zum Vorbild der Poesie wurde und wie ein (kunstvoller) Text dechiffriert werden musste. Bei den Romantikern erlebt das Motiv der Sprache der Natur einen semantischen Wandel, mit dem jedoch keine stilistischen Innovationen in Hinsicht der Sprachmittelauswahl einhergehen. Dank der Einfachheit der Naturbeschreibungen lassen sich Parallelen zu früheren Epochen und Schriften auffinden, die das Vorhandensein dieses Motivs belegen.

Hier tut sich aber auch ein weiteres Paradox auf: In der Prosa der Frühromantik ist das Buch der Natur zwar nicht mehr lesbar, aber die als göttliche Offenbarung geltenden Chiffren und Hieroglyphen der Natur sind als relativ konventionelle Tropen vorhanden. Die Autoren, die den Topos von der Sprache der Natur aufgreifen, nutzen dabei relativ unkomplizierte syntaktische Konstruktionen und typische rhetorische Tropen und Figuren, die scheinbar

83 Kurt Polycarp Joachim Sprengel, Versuch einer pragmatischen Geschichte der Arzneikunde, Bd. 3, Halle 1801, S. 436.

84 Ebd., S. 412.

85 Anadiplose ist eine rhetorische Figur und bedeutet „Wiederholung des Ausgangs eines Satzes [...] am Anfang des folgenden“. Anadiplose ist „Spezialfall der geminatio“, der Wortwiederholung, Wortverdoppelung. Siehe: Stefan Matuschek, Anadiplose [Artikel], in: G. Ueding, Historisches Wörterbuch der Rhetorik, Bd. 1, Tübingen 1992, S. 472.

86 Kurt Polycarp Joachim Sprengel, Versuch, Bd. 3, S. 412.

87 Andreas Kilcher, Das ästhetische Sprachparadigma der Kabbala, S. 298. 
im Widerspruch mit der komplizierten chiffrierten Mitteilung stehen. Zwar hört das Buch der Natur, die weiterhin als Text angesehen wird, auf, lesbar zu sein, und im Kontext der Weltund Selbsterkenntnis verliert der Topos seine primäre Klarheit. Dieser Krise aber begegnen die Autoren der Frühromantik mit einer pansemiotischen Vision des Ganzen.

\section{Literatur}

Alt, Peter-André, Aufklärung. Lehrbuch Germanistik, 2. Aufl., Stuttgart-Weimar 2001.

Al-Taie, Yvonne, Tropus und Erkenntnis: Sprach- und Bildtheorie der deutschen Frühromantik, Göttingen 2015.

Assmann, Aleida, Schriftspekulationen und Sprachutopien in Antike und früher Neuzeit, in: Eveline Goodman-Thau, Gert Mattenklott, Christoph Schulte (Hrsg.), Kabbala und Romantik. Die jüdische Mystik in der romantischen Geistesgeschichte, Tübingen 1994, S. 23-41.

Bader, Günter, Melancholie und Metapher: eine Skizze, Tübingen 1990.

Bär, Jochen A., Romantik [Artikel], in: Gert Ueding, Gregor Kalivoda u.a. (Hrsg.), Historisches Wörterbuch der Rhetorik, Bd. 8, Tübingen 2007, S. 333-362.

Bahr, Petra, Darstellung des Undarstellbaren. Religionstheoretische Studien zum Darstellungsbegriff bei A.G. Baumgarten und I. Kant, Tübingen 2004.

Billen, Josef, Hassel, Friedhelm, Undeutbare Welt. Sinnsuche und Entfremdungserfahrung in deutschen Naturgedichten von Andreas Gryphius bis Friedrich Nietzsche, Würzburg 2005.

Blumenberg, Hans, Die Lesbarkeit der Welt, 2. Aufl., Frankfurt a.M. 1983.

Campe, Rüdiger, Umbrüche und Wandlungen der Rhetorik, in: Horst Albert Glaser, György Mihály Vajda (Hrsg.), Die Wende von der Aufklärung zur Romantik 1760-1820: Epoche im Überblick, Amsterdam, Philadelphia, 2001, S. 589-612.

Feldmann, Doris, Jacobmeyer, Hannah, Logozentrismus [Artikel], in: Ansgar Nünning (Hrsg.), Metzler Lexikon Literatur- und Kulturtheorie: Ansätze - Personen - Grundbegriffe, 3. Aufl, Stuttgart 2004.

[Hamann, Johann, Georg] Johann Georg Hamann's Schriften und Briefe in vier Theilen. Vierter Theil, Hannover 1874.

Hamann, Johann, Georg, Sämtliche Werke. 6 Bände, hrsg. von J. Nadler, Bd. 1-2, Wien 1949-1957.

Hornung, Erik, „Hieroglyphisch denken“, Bild und Schrift im alten Ägypten in: Gottfried Boehm, Stephan E. Hauser (Hrsg.), Homo pictor, München, Leipzig 2001, S. 76- 86.

Höveler-Müller, Michael, Hieroglyphen lesen und schreiben. In 24 einfachen Schritten, München 2014.

[Johannes, Chrysostomus], Des heiligen Kirchenlehrers Johannes Chrysostomus Predigten und kleine Schriften, Bd. 1-9, Prag 1785.

Keil, Geert, Die naturalistische Herausforderung, in: ders., Kritik des Naturalismus, Berlin, New York 1993, S. 22-171.

Kemper, Dirk, Sprache der Natur, in: ders., Sprache der Dichtung. Wilhelm Heinrich Wackenroder im Kontext der Spätaufklärung, Stuttgart 1993, S. 172-181.

Kilcher, Andreas, Das ästhetische Sprachparadigma der Kabbala in der Romantik, in: ders., Die Sprachtheorie der Kabbala als ästhetisches Paradigma. Die Konstruktion einer ästhetischen Kabbala sein der Frühen Neuzeit, Stuttgart, Weimar 1998, S. 239-328. 
Krause, Peter D., Unbestimmte Rhetorik. Friedrich Schlegel und die Redekunst um 1800 [Reihe: Rhetorik-Forschungen, hrsg. von Joachim Dyck, Walter Jens, Gert Ueding, Bd. 14], Tübingen 2001.

Kupfer, Alexander, Die Hieroglyphensprache der Natur, in: ders., Die künstlichen Paradiese: Rausch und Realität seit der Romantik. Ein Handbuch. Sonderausgabe. Stuttgart, Weimar 2006, S. $162-168$.

Leusing, Reinhard, Die Stimme als Erkenntnisform - zu Novalis‘ Roman „Die Lehrlinge zu Sais“, Stuttgart 1993.

Ohly, Friedrich, Neue Zeugen des „Buchs der Natur“ aus dem Mittelalter, in: Hagen Keller, Nikolaus Staubach (Hrsg.), Iconologia sacra. Mythos, Bildkunst und Dichtung in der Religions- und Sozialgeschichte Alteuropas [Arbeiten zur Frühmittelalterforschung. Schriftenreihe des Instituts für Frühmittelalterforschung der Universität Münster, Bd. 23], Berlin, New York 1994, S. 546-568.

Matuschek, Stefan, Anadiplose [Artikel], in: G. Ueding, Historisches Wörterbuch der Rhetorik, Bd. 1, Tübingen 1992, S. 472.

Novalis, Die Lehrlinge zu Sais, in: Novalis, Werke, herausgegeben und kommentiert von Gerhard Schulz, München 1969.

Novalis, Werke. Tagebücher und Briefe Friedrich von Hardenbergs, hrsg. von H.-J. Mähl, R. Samuel, Bd. 2, München 1978.

Nöth, Winfried, Ökosemiotik, in: ders., Handbuch der Semiotik, 2., vollständig neu bearbeitete und erweiterte Aufl., Stuttgart, Weimar 2000, S. 250-253.

Pernkopf, Elisabeth, Alphabetisierte Natur? Sprachformen in den Naturwissenschaften, in: Reinhold Esterbauer, Elisabeth Pernkopf, Marion Schonhart (Hrsg.), Spiel mit der Wirklichkeit: zum Erfahrungsbegriff in den Naturwissenschaften, Würzburg 2004, S. 81-94.

Renger, Almut-Barbara, Hieroglyphe [Artikel], in: Günter Butzer, Joachim Jacob (Hrsg.), Metzler Lexikon literarischer Symbole, 2. Aufl., Stuttgart 2012, S. 181-182.

Sauder, Gerhard, Aufklärerische Bibelkritik und Bibelrezeption in Goethes Werk, in: „GoetheJahrbuch“ 2001, Bd. 118, S. 108-125.

Schanze, Helmut, Romantische Rhetorik, in: ders. (Hrsg.), Romantik-Handbuch, Stuttgart 1994, S. 338-339.

Schanze, Helmut, Romantik und Rhetorik, Rhetorische Komponenten der Literaturprogrammatik um 1800, in: ders. (Hrsg.), Rhetorik. Beiträge zu ihrer Geschichte in Deutschland vom 16.-20. Jahrhundert, Frankfurt a.M. 1974, S. 126-144.

Schmitz-Emans, Monika, Entzifferung, Buchstabieren und Konjektur: Aspekte und Funktionen des Weltschriftgleichnisses bei Georg Christoph Lichtenberg, in: Jahrbuch der Lichtenberg-Gesellschaft 1991, S. 29-58.

Schmitz-Emans, Monika, Buchstabe [Artikel], in: Günter Butzer, Joachim Jacob (Hrsg.), Metzler Lexikon literarischer Symbole, 2. Aufl., Stuttgart 2012, S. 67-68.

Schmitz-Emans, Monika, Romantische Sprachästhetik, in: Horst Albert Glaser, György Mihály Vajda (Hrsg.), Die Wende von der Aufklärung zur Romantik 1760-1820: Epoche im Überblick, Amsterdam, Philadelphia 2001, S. 567-587.

Simon, Ralf, Die Bildlichkeit des lyrischen Textes. Studien zu Hölderlin, Brentano, Eichendorff, Heine, Mörike, George und Rilke, München 2011.

Speer, Andreas, Die entdeckte Natur. Untersuchungen zu begründungsversuchen einer ,scientia naturalis`im 12. Jahrhundert, Leiden, New York, Köln 1995. 
Sprengel, Kurt, Polycarp, Joachim, Versuch einer pragmatischen Geschichte der Arzneikunde, Bd. 3, Halle 1801.

Veldhuis, Henri, Ein versiegeltes Buch. Der Naturbegriff in der Theologie J.G. Hamanns (1730-1788). Aus dem Niederländ. übers. von Renate Drewes-Siebel, Berlin, New York 1994.

Wackenroder, Wilhelm, Heinrich, Werke und Briefe, Berlin, München 1984.

Weimar, Klaus u.a. (Hrsg.), Reallexikon der deutschen Literaturwissenschaft: Neubearbeitung des Reallexikons der deutschen Literaturgeschichte, Bd. 1: A-G, Berlin, New York 2007.

Zollna, Isabel, Chiffre [Artikel], in: Gert Ueding, Gregor Kalivoda u.a. (Hrsg.), Historisches Wörterbuch der Rhetorik, Bd. 2, Tübingen 1994, S. 173-174. 